\title{
Ringraziamenti ai reviewer
}

$\grave{E}$ noto e riconosciuto che il livello scientifico di una rivista è dato dalla qualità dei contributi e dalla competenza degli autori, ma anche dal rigore del processo di referaggio. Su questo presupposto Mecosan da oltre 25 anni ha fondato il proprio prestigio nel mondo accademico e nel mondo degli operatori. Il processo di referaggio non è mai semplice perché richiede tempo, conoscenze, impegno dei reviewer che sono chiamati a un processo di valutazione che consiste soprattutto nel dare agli autori suggerimenti sulle modalità per migliorare i propri contributi. Tra autori e reviewer si attiva un processo bidirezionale mediato dall'editor e dal principio di double blind, che garantisce imparzialità, neutralità e autonomia di giudizio. Tra l'altro i reviewer sono sottoposti a una duplice pressione, quella del tempo, che deve essere breve per consentire agli autori di avere risposte alle proprie attese, e quella degli impegni connessi alle proprie attività istituzionali. Per questo esprimo il più vivo ringraziamento ai reviewer di seguito citati a nome del Comitato scientifico, del Comitato di Redazione, dei co-editor e mio personale. Da parte mia mi scuso se qualche volta ho fatto pressioni, sempre mosso dalla finalità di migliorare Mecosan come bene comune della comunità scientifica di chi si occupa di economia e management applicato al sistema di tutela della salute inteso in senso ampio. Per il futuro mi impegno a rendere ancora più agevole il lavoro dei reviewer.

Elio Borgonovi

Per l'annata 2018 si ringraziano: Alberto Ricci (Università Bocconi); Andrea Francesconi (Università di Trento); Andrea Garlatti (Università degli Studi di Udine); Anna Prenestini (Università Bocconi); Antonio Botti (Università degli Studi di Salerno); Carmela Annarumma (Università degli Studi di Salerno); Davide Galli (Università Cattolica di Milano); Enzo Bivona (Università di Palermo); Federica Bandini (Università di Bologna); Federico Lega (Università degli Studi di Milano Statale); Gabriella Piscopo (Università degli Studi di Salerno); Giuseppe Marcon (Università Ca' Foscari di Venezia); Luca Del Bene (Università Politecnica delle Marche); Maddalena Sorrentino (Università degli Studi di Milano); Manuela Brusoni (Università Bocconi); Manuela Macinati (Università Cattolica di Milano); Mara Bergamaschi (Università degli Studi di Bergamo); Marco Morelli (Università del Piemonte Orientale); Marta Marsilio (Università di Trento); Paola Briganti (Università degli Studi di Napoli Parthenope); Paola Orlandini (Università degli Studi di Milano-Bicocca); Paolo Ricci (Università degli Studi di Napoli Federico II); Pierluigi Catalfo (Università degli Studi di Catania); Rocco Palumbo (Università di Salerno); Stefania De Simone (Istituto di Ricerca su Innovazione e Servizi per lo Sviluppo); Stefano Calciolari (Università della Svizzera Italiana); Stefano Villa (Università Cattolica di Milano); Valeria Rappini (Università Bocconi); Valeria Tozzi (Università Bocconi). 